\title{
Characteristic Classes and Quadric Bundles
}

\author{
Dan Edidin* and William Graham ${ }^{\dagger}$ \\ Department of Mathematics \\ University of Chicago \\ Chicago IL 60637
}

\section{Introduction}

In this paper we construct Stiefel-Whitney and Euler classes in Chow cohomology for algebraic vector bundles with nondegenerate quadratic form. These classes are not in the algebra generated by the Chern classes of such bundles and are new characteristic classes in algebraic geometry. On complex varieties, they correspond to classes with the same name pulled back from the cohomology of the classifying space $B S O(N, \mathbb{C})$. The classes we construct are the only new characteristic classes in algebraic geometry coming from the classical groups ([T2], [EG]).

We begin by using the geometry of quadric bundles to study Chern classes of maximal isotropic subbundles. If $V \rightarrow X$ is a vector bundle with quadratic form, and if $E$ and $F$ are maximal isotropic subbundles of $V$ then we prove (Theorem 1) that $c_{i}(E)$ and $c_{i}(F)$ are equal mod 2. Moreover, if the rank of $V$ is $2 n$, then $c_{n}(E)= \pm c_{n}(F)$, proving a conjecture of Fulton. We define Stiefel-Whitney and Euler classes as Chow cohomology classes which pull back to Chern classes of maximal isotropic subbundles of the pullback bundle. Using the above theorem we show (Theorem 2) that these classes exist and are unique, even though $V$ need not have a maximal isotropic subbundle. These constructions also make it possible to give "Schubert" presentations,

\footnotetext{
*Partially supported by an NSF postdoctoral fellowship

${ }^{\dagger}$ Partially supported by an NSF postdoctoral fellowship
} 
depending on a fixed maximal isotropic flag, of the Chow rings of quadric and isotropic flag bundles. The presentation for the flag bundle is closely

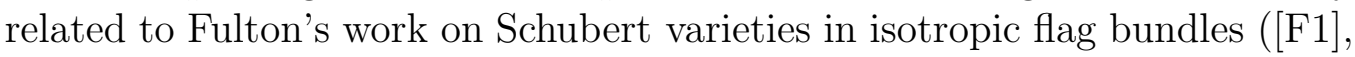
[F2]).

An interesting aspect of this work is that it emphasizes the difference between principal $S O(N)$ bundles in the Zariski and étale topologies. As is well known (Sem-Chev), the group $S O(N)$, unlike $S L(N)$, is not special; i.e. not all principal bundles which are locally trivial in the étale topology are locally trivial in the Zariski topology. This fact manifests itself as follows. The Stiefel-Whitney classes in $A^{*}(X ; \mathbb{Z} / 2 \mathbb{Z})$ we construct are for bundles which are locally trivial in the Zariski topology. In the étale topology it is not always possible to construct these classes, as there are bundles for which the corresponding topological Stiefel-Whitney classes are not algebraic [T1]. Similarly, we construct an integral Euler class for Zariski locally trivial $S O(2 n)$ bundles, but for general $S O(2 n)$ bundles only a power of 2 times the Euler class is integral [T1].

The paper is organized as follows. Section 11 is the introduction. Section 2 is largely a collection of standard and/or easy facts about vector bundles with quadratic form, quadric bundles and isotropic flag bundles which we use and which have not been adequately presented in the literature.

The remainder of the paper is new. In Section 3 we prove the theorem on Chern classes of maximal isotropic subbundles, including Fulton's conjecture. Section 1 contains the construction of Stiefel-Whitney and Euler classes for bundles with quadratic form which are locally trivial in the Zariski topology. In Section 5 we show that for smooth complex varieties the classes we construct map to the classes with the same names in singular cohomology.

Section 6 deals with bundles whose structure group reduces to $S O(2 n)$ but which are not locally trivial. In particular, we show that there is a characteristic class $y_{n} \in A^{n} X$ such that $\frac{1}{2^{n-1}} y_{n}$ is an Euler class. We also give a natural (with $\mathbb{Z}\left[\frac{1}{2}\right]$ coefficients) presentation of the Chow ring of the quadric bundle as an algebra with two generators and two relations, such that the Euler and Chern classes of $V$ are the coefficients in the relations. This presentation is the $S O(2 n)$ analogue of the presentation of $A^{*} \mathbb{P}(V)$ over $A^{*} X$, where the Chern classes of $\mathrm{V}$ are the coefficients in the sole relation. Viewing the isotropic flag variety $F l(V)$ as a tower of quadrics bundles, we also describe $A^{*}\left(F l(V) ; \mathbb{Z}\left[\frac{1}{2}\right]\right)$. Finally, in Section 7 we give a "Schubert" presenations for $A^{*} Q$ and $A^{*}(F l(V))$, which depend on a fixed maximal isotropic 
subbundle $F \subset V$.

Acknowledgements. We would like to thank William Fulton for his generous help, in particular for informing us of his conjecture and suggesting that the Euler class was related to top Chern classes of maximal isotropic subbundles. Some of the facts presented in Section 2 are based on his lectures at the University of Chicago. We are also grateful to Burt Totaro for his example showing that the Euler class need not be integral for bundles which are not locally trivial in the Zariski topology.

\section{Preliminaries}

Throughout this paper, unless otherwise noted, all schemes are assumed to be of finite type over an arbitrary field.

Operational Chow groups If $X$ is a scheme, then $A_{*} X$ denotes the Chow homology groups defined in [Fulton, Chapter 1], and $A^{*} X$ is the operational Chow cohomology ring of [Fulton, Chapter 17]. An element $c \in A^{p} X$ is a collection of homomorphisms $A_{*} X^{\prime} \rightarrow A_{*-p} X^{\prime}$ for all $X^{\prime} \rightarrow X$ which is compatible with proper pushforward, flat pull-back, and interstions. By construction, if $X \stackrel{f}{\rightarrow} Y$ is any map there is always a ring homomorphism $f^{*}: A^{*} Y \rightarrow A^{*} X$. If $X$ is regular, then $A^{*} X$ is just the usual Chow groups with multiplication given by intersection product. Now if $R$ is any coefficient ring, we can also define $A^{*}(X ; R)$ as maps from $A_{*} X^{\prime} \otimes R \rightarrow A_{*} X^{\prime} \otimes R$ for all $X^{\prime} \rightarrow X$ satisfying the same conditions as above. There is always a homomorphism $A^{*} X \otimes R \rightarrow A^{*}(X ; R)$. If $X$ admits a resolution of singularities then standard arguments (cf. [K]) show that $X$ is an isomorphism.

Quadratic Forms A quadratic form $q$ on a vector space $V$ will always mean a hyperbolic quadratic form: in other words, we assume that $V$ (of dimension $2 n$ or $2 n+1$ ) has an isotropic subspace of dimension $n$ (if the ground field is algebraically closed of characteristic not equal to 2 , this is automatically satisfied). We can choose a basis $v_{1}, v_{2}, \ldots$ of $V$ such that the quadratic norm $q(v, v)$ of a vector $v=\sum x_{i} v_{i}$ is given by

$$
\begin{gathered}
q(v, v)=x_{1} x_{2 n}+\ldots+x_{n} x_{n+1}(\text { if } \operatorname{dim} V=2 n) \\
q(v, v)=x_{1} x_{2 n+1}+\ldots+x_{n} x_{n+2}+a x_{n+1}^{2}(\text { if } \operatorname{dim} V=2 n+1)
\end{gathered}
$$


where $a$ is a nonzero scalar. The reason our form must be hyperbolic is that otherwise there may be no nonzero isotropic vectors in $V$. For example, there are no nonzero isotropic vectors for $\mathbb{R}^{2}$ with quadratic form $x_{1}^{2}+x_{2}^{2}$. While the theorems we prove are for schemes of arbitrary fields, we will abuse notation and refer to the group that preserves a quadratic form on a $N$ dimensional vector space as $O(N)$, even though this notation is only correct when the field is algebraically closed.

Let Iso $(V)$ be the variety of maximal isotropic subspaces of $V$. The groups $S O(N)$ and $O(N)$ act on $\operatorname{Iso}(V)$. The $O(N)$ action is always transitive, but the $S O(N)$ action is only transitive if $N$ is odd. If $N$ is even, there are two $S O(N)$ orbits and two maximal isotropic subspaces $E$ and $F$ of $V$ lie in the same $S O(N)$ orbit if and only if $\operatorname{dim} E \cap F \equiv n(\bmod 2)$. We will say that $E$ and $F$ are in the same family if they are in the same $S O(N)$ orbit, and in opposite families otherwise.

Let $F l(V)$ denote the flag variety of complete isotropic flags of a certain length in $V$. This length is $n$ if $\operatorname{dim} V=2 n+1$ and $n-1$ if $\operatorname{dim} V=2 n$. If $\operatorname{dim} V=2 n$ we let $F l_{n}(V)$ denote the variety of isotropic flags of length $n$. A point in one of these varieties corresponds to a flag $E_{1} \subset E_{2} \subset \ldots$ of isotropic subspaces of $V$ (where $\operatorname{dim} E_{i}=i$ ). In the even dimensional case, every length $n-1$ isotropic flag can be completed to exactly two isotropic length $n$ flags, and the space $F l_{n}(V)$ is a disconnected double cover of $V$. Again, the groups $S O(N)$ and $O(N)$ act. The $S O(N)$ action on $F l(V)$ is transitive, but there are two $S O(2 n)$ orbits on $F l_{n}(V)$; two length $n$ isotropic flags $E$. and $F$. are in the same $S O(N)$ orbit if and only if $\operatorname{dim} E_{n} \cap F_{n} \equiv n(\bmod 2)$.

Let $Q \subset \mathbb{P}(V)$ ] denote the quadric of isotropic vectors. If $E$ and $F$ are maximal isotropic subspaces then $\mathbb{P}(E)$ and $\mathbb{P}(F)$ are subvarieties of $Q$ called rulings. $\mathbb{P}(E)$ and $\mathbb{P}(F)$ represent the same class in $A_{n-1}(Q)$ if and only if $E$ and $F$ lie in the same $S O(N)$ orbit. Thus, if $\operatorname{dim} V=2 n$, there are two families of rulings giving rise to two Chow classes $e$ and $f$, and, letting $h$ denote the hyperplane section, we have $h^{n-1}=e+f$. If $\operatorname{dim} V=2 n+1$ there is only one family and $h^{n}=2 e$.

Lemma 1 (a) If $N=2 n$ is even, then a basis for $A_{*}(Q)$ is $1, h, h^{2}, \ldots, h^{n-1}, e, h e, \ldots, h^{n-1} e$. (b) If $N=2 n+1$ is odd, then a basis for $A_{*}(Q)$ is $1, h, h^{2}, \ldots, h^{n-1}, e, h e, \ldots, h^{n-1} e$.

\footnotetext{
${ }^{1}$ Throught this paper $\mathbb{P}(V)$ will denote the scheme of lines in $V$.
} 
Remark: Because a quadric has a decomposition into affine cells, $A_{*}(U \times$ $Q)=A_{*}(U) \otimes A_{*}(Q)$. This fact will be essential when we compute the Chow rings of quadric bundles.

Proof: (a) In any characteristic the hyperbolic quadric has an affine cellular decompostion with 1 cell in every dimension, except 2 cells in dimension $n-1$. Hence $A_{i}(Q)=\mathbb{Z}$ for $i \neq n-1$, and $A_{n-1}(Q)=\mathbb{Z} \oplus \mathbb{Z}$. Since $e$ is a class of a linear space, $h^{n-1} e=1$. Hence, for $i \leq n-1, h^{i}$ is a generator of $A_{n-i}(Q)$. Likewise, the $h^{i} e$ are also generators of $A_{i}(Q)$ for $i \leq n-1$. This proves (a). The proof of (b) is similar. $\triangle$

In the sequel we use the following relations in the Chow ring of an evendimensional quadric.

Lemma 2 Let $Q \subset \mathbb{P}^{2 n-1}$ be an even-dimensional quadric. Then $e^{2}=f^{2}=0$ and $e f=1$ if $n$ is even, and $e^{2}=f^{2}=1$ and $e f=0$ if $n$ is odd.

Proof: First note that $e$ and $f$ both have degree 1 , so $h^{n-1} e=h^{n-1} f=1$. Since $h^{n-1}=e+f$, it follows that $e^{2}=f^{2}$. Also, since $h^{2 n-2}=(e+f)^{2}=2$, it follows either that $e^{2}=f^{2}=1$ and $e f=0$, or, $e^{2}=f^{2}=0$ and ef $=1$. The linear system spanned by $e$ is an $S O(2 n)$ orbit of isotropic $n$-planes. One such $n$-plane is given by $x_{1}=x_{2}=\ldots=x_{n}=0$. When $n$ is even, this plane can be taken to the plane $x_{n+1}=x_{n+2}=\ldots=x_{2 n}=0$ via an element of $S O(2 n)$ (an even permutation). Projectivizing, we see that there are two disjoint elements in the linear system spanned by $e$. Hence $e^{2}=0$.

When $n$ is odd, the same argument shows that there are elements in the linear system corresponding to $e$ that are disjoint from elements of $f$. Thus $e f=0$.

The constructions above also work for vector bundles. We adopt the convention that a quadratic form $q$ on a vector bundle $V \rightarrow X$ means a section of $S^{2}\left(V^{*}\right)$ which restricts to a nondegenerate hyperbolic quadratic form on each fiber. The constructions of $Q, F l(V)$, and $F l_{n}(V)$ all carry over to the bundle case. A maximal isotropic subbundle is defined to be an isotropic subbundle of rank $n$ (where the rank of $V$ is $2 n$ or $2 n+1$ ). If the rank of $V$ is even and the structure group reduces to $S O(2 n)$ (this is automatically satisfied in the Zariski locally trivial case - see below) then the pullback of $V$ to $F l(V)$ has two tautological maximal isotropic subbundles. If the rank 
is odd then there is only one tautological maximal isotropic subbundle over $F l(V)$. These facts will be used extensively in the sequel.

The description of $S O(2 n)$ orbits of maximal isotropic subspaces yields the following fact about maximal isotropic subbundles.

Proposition 1 If $E$ and $F$ are maximal isotropic subbundles of $V \rightarrow X$, where the rank of $V$ is even, then $\operatorname{dim} E_{x} \cap F_{x}$ is constant $\bmod 2$ on each component of $X$.

We say the pair $(V, q)$ is Zariski locally trivial (or simply locally trivial) if there exists a Zariski open covering $\left\{U_{i}\right\}$ of $X$, such that $V_{\mid U_{i}} \simeq X \times \mathbb{A}^{N}$ and the under the isomorphisms the quadratic norm on a fiber over $u \in U_{i}$ is given by:

$$
\begin{gathered}
(\operatorname{rank} V=2 n) q(v, v)=v_{1} v_{2 n}+\ldots+v_{n} v_{n+1} \\
(\operatorname{rank} V=2 n+1) q(v, v)=v_{1} v_{2 n+1}+\ldots+v_{n} v_{n+2}+f(u) v_{n+1}^{2} .
\end{gathered}
$$

Here $v=\left(v_{1}, \ldots, v_{N}\right)$ is a vector in the fiber over $u$, and $f$ is a nowhere vanishing function on $U_{i}$. (Swan $[\mathbf{S}$, Cor 1.2] has shown that any $(V, q)$ is locally trivial in the étale topology; i.e., there is an étale cover $\left\{U_{i} \stackrel{\pi_{i}}{\rightarrow} X\right\}$ such that $\left(\pi_{i}^{*} V, \pi_{i}^{*} q\right)$ is as above).

Remarks: Not all bundles with quadratic form are locally trivial in the Zariski topology, as the following example shows. Example: Let $X=\mathbb{C}^{*}$ be the 1 dimensional torus. Let $V=X \times \mathbb{C}^{2}$ be a trivial rank two vector bundle. Define a quadratic form on $V$ by the rule that over a point $\lambda \in \mathbb{C}^{*}$ the quadratic norm is $v_{1}^{2}+\lambda v_{2}^{2}$. The pair $(V, q)$ is locally trivial in the étale topology but not in the Zariski topology.

Maximal isotropic subbundles need not exist, even if $V$ is Zariski locally trivial; we give a simple example in Section 6. However, if $V$ has a maximal isotropic subbundle, then the pair $(V, q)$ is locally trivial. If the rank of $V$ is odd, then it is not always possible to choose trivializations so that the functions $f(u)$ above are identically 1 , even if $V$ has a maximal isotropic subbundle. For instance, if $V$ is a self-dual line bundle, then the trivializations can be so chosen if and only if $V$ is the trivial line bundle.

If the pair $(V, q)$ is locally trivial in the Zariski topology, then so is the associated quadric bundle. This assertion is obvious if the rank is even. In the odd rank case, it suffices to show that if $V \rightarrow U$ is a trivial vector bundle 
with quadratic form as above, then the quadric bundle $Q$ on $U$ is isomorphic to $U \times Q_{p t}$, where $Q_{p t}$ is the quadric over a point. The desired isomorphism maps $u \times\left(v_{1}, \ldots, v_{2 n+1}\right)$ to $\left(f(u) v_{1}, \ldots, f(u) v_{n}, v_{n+1}, \ldots, v_{2 n+1}\right)$.

Note that if $(V, q)$ is Zariski locally trivial of rank $2 n$ then the structure group of $V$ reduces to $S O(2 n)$. The reason is that the principal bundle is also Zariski locally trivial. This bundle is a priori an $O(2 n)$ bundle, but any Zariski locally trivial bundle on a connected space has the same number of components as the fiber. Thus (assuming $X$ connected) the $O(2 n)$ principal bundle has two components, implying that the structure group reduces. If the rank is $2 n+1$ we cannot conclude that the structure group reduces to $S O(2 n+1)$, because the associated $O(2 n+1)$ principal bundle need not be Zariski locally trivial (since the function $f(u)$ need not be identically 1 ).

The following lemma well be needed in the sequel.

Lemma 3 Let $f: Y \rightarrow X$ be a bundle with complete fibers which is locally trivial in the Zariski topology. Then $f^{*}$ is injective for Chow cohomology with any coefficients.

Proof: Because $Y$ is a locally trivial in Zariski topology, $f: Y \rightarrow X$ is a proper Chow envelope in the sense of [Fulton, Chapter 18]. The lemma

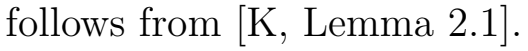

Remark on characteristic 2. The results of this paper hold in characteristic 2 (and if we assume that the constant $a$ in the odd rank quadratic form is a square in the field of definition). The reason is that the integral quadrics $x_{1} x_{2 n}+\ldots x_{n-1} x_{n+1}$ or $x_{1} x_{2 n+1}+\ldots x_{n} x_{n+2}+x_{n+1}^{2}$ are smooth over $S p e c \mathbb{Z}$. Likewise we can construct an isotropic flag variety which is smooth over $S p e c \mathbb{Z}$. By specializing from characteristic 0, we can prove all the necessary intersection theoretic properties of the quadric and flag varieties defined over $\mathbb{F}_{2}$. Since our constructions depend only on intersection theory, the results follow. For consistency of notation we take $S O(N)$ in characteristic 2 to mean the connected component of the identity in $O(N)$.

Note that in characteristic 2 , even if the field is algebraically closed not all forms are hyperbolic. For example the quadratic form $x_{1}^{2}+x_{2}^{2}$ over $\overline{\mathbb{F}}_{2}$ is not hyperbolic. 


\section{Chern Classes of Isotropic Subbundles}

The purpose of this section is to prove the following theorem. Part (c) is Fulton's original conjecture.

Theorem 1 Let $V$ be a vector bundle on a connected scheme $X$, of rank $N$ equal to $2 n$ or $2 n+1$, equipped with a non-degenerate quadratic form, and let $d=N-n$. Let $E$ and $F$ be maximal isotropic subbundles of $V$.

(a) There exist classes $c_{i}$ and $d_{i}$ in $A^{*}(X)$ such that $2 c_{i}=c_{i}(E)+c_{i}(F)$ and $2 d_{i}=c_{i}(V / E)+c_{i}(V / F)$. These classes are functorial for maps $X^{\prime} \rightarrow X$.

(b) If $E$ and $F$ are in the same (resp. opposite) families, then $c_{d}(V / E)=$ $c_{d}(V / F)\left(\right.$ resp. $\left.-c_{d}(V / F)\right)$.

(c) If the rank of $V$ is $2 n$, then $c_{n}(E)=c_{n}(F)$ if $E$ and $F$ are in the same family, and $c_{n}(E)=-c_{n}(F)$ if $E$ and $F$ are in opposite families.

Proof: Note that (c) follows immediately from (b), because in the even rank case, we may identify $V / E$ and $V / F$ with $E^{*}$ and $F^{*}$ respectively.

If $Y \rightarrow X$, then $c_{i}(V / E) \cap[Y]$ and $c_{i}(V / F) \cap[Y]$ are both supported in $A_{*}(Y)$. Replacing $X$ with $Y$ it suffices to check the relations after capping with $[X]$. In the remainder of the proof, we will use the notations $c_{i}(V / E)$, etc.,to mean $c_{i}(V / E) \cap[X] \in A_{*}(X)$. Let $Q \rightarrow X$ be the quadric bundle associated to the quadratic form on $V$. Let $i: Q \hookrightarrow \mathbb{P}(V)$ be the inclusion, and let $\pi: \mathbb{P}(V) \rightarrow X$ and $\rho: Q \rightarrow X$ be the projections. Because $E$ and $F$ are isotropic subbundles, $\mathbb{P}(E)$ and $\mathbb{P}(F)$ are subvarieties of $Q$.

Now we will examine $[\mathbb{P}(E)]$ and $[\mathbb{P}(F)]$ in the Chow groups of $Q$. Set $\gamma=[\mathbb{P}(E)]$, and let $h$ be the pullback to $A_{*}(Q)$ of the hyperplane class $H$ of $\mathbb{P}(V) \rightarrow X$. Since $\mathbb{P}(E) \subset Q$ is a regular embedding we can define a bivariant class $\gamma \in A^{*}(Q \rightarrow X)$ by the formula $\alpha \rightarrow \gamma \cap \rho^{*} \alpha \in A_{*}(Q)$.

Lemma 4 Every $\beta \in A_{*}(Q)$ can be written as

$$
\sum_{i=0}^{i=n-1} h^{i} \rho^{*}\left(\alpha_{i}\right)+\sum_{i=0}^{i=n-1} h^{i} \gamma \cap \rho^{*}\left(\beta_{i}\right) .
$$

Proof of Lemma 4: By Lemma 1, the classes listed generate the Chow groups of the fibers. Because $V$ has a maximal isotropic subbundle, $Q \rightarrow X$ is locally trivial in the Zariski topology. The conclusion then follows by Noetherian induction as in [Fulton, Proposition 1.9]. 
We now finish the proof of the theorem. First suppose that the $E$ and $F$ are in the same family (this is automatically satisfied in the odd rank case). Then $[\mathbb{P}(E)]$ and $[\mathbb{P}(F)]$ agree on each fiber of $Q$. Thus in $A_{*}(Q)$,

$$
[\mathbb{P}(E)]-[\mathbb{P}(F)]=\sum_{i>0} h^{d-1-i} \cap \rho^{*}\left(\alpha_{i}\right),
$$

for some classes $\alpha_{i}$ in $A_{*}(X)$. But

$$
i_{*}\left(h^{j} \cap \rho^{*} \alpha\right)=c_{1}(\mathcal{O}(Q)) \cap H^{j} \cap \pi^{*}(\alpha) .
$$

Since the quadric bundle corresponds to a section of $\mathcal{O}_{\mathbb{P}(V)}(2), c_{1}(\mathcal{O}(Q))=$ $2 H$. Thus

$$
i_{*}([\mathbb{P}(E)]-[\mathbb{P}(F)])=\sum_{i>0} 2 H^{i} \cap \pi^{*}\left(\alpha_{d-i}\right) .
$$

On the other hand, [Fulton, Example 3.2.17] implies that

$$
i_{*}([\mathbb{P}(E)]-[\mathbb{P}(F)])=\sum_{i \geq 0} H^{i} \cap \pi^{*}\left(c_{d-i}(V / E)-c_{d-i}(V / F)\right) .
$$

Comparing these expressions, we see that $c_{d}(V / E)=c_{d}(V / F)$, which proves part (b). Moreover, defining $d_{i}=\alpha_{i}+c_{i}(V / F)$, we see that $2 d_{i}=c_{i}(V / E)+$ $c_{i}(V / F)$ as desired. In the even case, to get the classes $c_{i}$, we simply define $c_{i}=(-1)^{i} d_{i}$. In the odd case, we no longer have $V / E$ and $V / F$ isomorphic to $E^{*}$ and $F^{*}$. However, an easy computation (using the fact that $c_{1}(V)=$ $\left.c_{1}\left(E^{\perp} / E\right)=c_{1}\left(F^{\perp} / F\right)\right)$ shows that

$$
c\left(E^{*}\right)+c\left(F^{*}\right)=\frac{c(V / E)+c(V / F)}{1+c_{1}(V)} .
$$

Therefore, if we define the $c_{i}$ by the formula

$$
\sum_{i=0}^{n}(-1)^{i} c_{i}=\frac{\sum_{i=0}^{n} d_{i}}{1+c_{1}(V)} .
$$

we get $2 c_{i}=c_{i}(E)+c_{i}(F)$, as desired. The classes $c_{i}$ and $d_{i}$ are obviously functorial for maps $X^{\prime} \rightarrow X$.

This proves the theorem in the case where $E$ and $F$ are in the same family. In the case where $E$ and $F$ are in opposite families we compare $[\mathbb{P}(E)]$ and $h^{n-1}-[\mathbb{P}(F)]$ to obtain the result. 


\section{Construction of Euler and Stiefel-Whitney Classes}

Let $V \rightarrow X$ be a vector bundle of rank $N=2 n$ or $N=2 n+1$ with a nondegenerate quadratic form $q: V \otimes V \rightarrow \mathcal{O}$.

Definition 1 (a) A Stiefel-Whitney class for $V \rightarrow X$ is a class $w_{2 i} \in$ $A^{i}(X ; \mathbb{Z} / 2 \mathbb{Z})$ such that for any $f: Y \rightarrow X$ and any maximal isotropic subbundle $E \subset f^{*} V$ we have $f^{*} w_{2 i} \equiv c_{i}(E)(\bmod 2)$

(b) If the rank of $V$ equals $2 n$, then an Euler class for $V \rightarrow X$ is a class $x \in A^{n}(X)$ such that for any $f: Y \rightarrow X$, and any maximal isotropic subbundle $E \subset f^{*} V$ we have $f^{*} x= \pm c_{n}(E)$.

The purpose of this section is to prove the existence of these classes when the pair $(V, q)$ is locally trivial in the Zariski topology. For the remainder of this section we will therefore assume that $(V, q)$ is Zariski locally trivial. We may repeat this assumption for emphasis.

Remark. Note that if $E \subset V$ is a maximal isotropic subbundle, then $c_{n}(E)$ is an Euler class for $V$. In Section 5 we will explain the connnection between our defintions and those given in topology.

The next proposition states some basic properties of Stiefel-Whitney and Euler classes.

Proposition 2 (1) Pullbacks of Stiefel-Whitney (resp. Euler) classes are Stiefel-Whitney (resp. Euler) classes for the pullback bundle.

(2) Stiefel-Whitney classes are unique.

(3) An Euler class is unique up to sign; i.e., if $x, y$ are both Euler classes for a bundle $V_{n}$, then $x= \pm y$.

(4) If $x$ is an Euler class of $V_{n}$, then $x^{2}=(-1)^{n} c_{2 n}\left(V_{n}\right)$.

Proof: Property (1) follows from the definition. Next consider the map $f$ : $F l(V) \rightarrow X$. The bundle $f^{*} V$ has a maximal isotropic subbundle $E$. Suppose $w_{2 i}$ and $w_{2 i}^{\prime}$ are Stiefel-Whitney classes in degree $A^{i}(X)$. By definition,

$$
f^{*} w_{2 i}=f^{*} w_{2 i}^{\prime}=c_{i}(E)(\bmod 2) .
$$

Since $F l(V) \rightarrow X$ is locally trivial, $f^{*}$ is injective (Lemma 3), so $w_{2 i}=w_{2 i}^{\prime}$ in $A^{*}(X ; \mathbb{Z} / 2 \mathbb{Z})$, proving (2). Likewise, $f^{*} x$ and $f^{*} y$ are equal to $\pm c_{n}\left(V_{n}^{\prime}\right)$, so $x= \pm y$ as Chow cohomology classes. This proves (3). 
Finally, note that, via the quadratic form, we can identify $V_{n}^{* *}=f^{*} V_{n} / V_{n}^{\prime}$. Thus,

$$
c_{2 n}\left(f^{*} V_{n}\right)=c_{n}\left(V_{n}^{\prime}\right) c_{n}\left(V_{n}^{* *}\right)=(-1)^{n} f^{*}\left(x^{2}\right) .
$$

Since $f^{*}$ is injective, (4) follows. $\triangle$

Theorem 2 Let $V \rightarrow X$ be a vector bundle with a nondegenerate quadratic form $q$. If the pair $(V, q)$ is locally trivial in the Zariski topology then $V$ has Stiefel-Whitney classes $w_{2 i} \in A^{i}(X ; \mathbb{Z} / 2 \mathbb{Z})$. If the rank of $V$ is $2 n$, then $V$ has two Euler classes $x_{n}$ and $-x_{n}$ in $A^{n}(X)$.

Remark 1. Proposition 2 and Theorem 2 together show that Stiefel-Whitney and Euler classes are characterstic classes for vector bundles with quadratic form which are locally trivial in the Zariski topology.

Remark 2. In topology the sign of the Euler class is determined by the choice of an orientation of the bundle, or in other words, a reduction of structure group to $S O(N)$. The same holds in the algebraic case. This is equivalent to choosing a maximal isotropic subbundle of the pullback of $V$ to the flag bundle.

Proof: Consider the Cartesian diagram

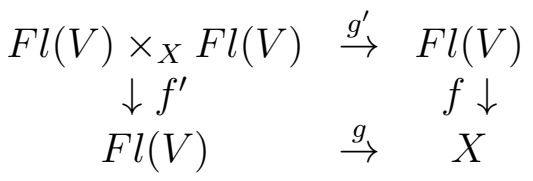

Let $E \subset f^{*} V$ be one of the two tautological maximal isotropic subbundles. (The choice of $E$ is equivalent to choosing an orientation.) To show that $w_{2 i}$ exists we must show that $c_{i}(E)(\bmod 2)$ is the pullback $(\bmod 2)$ of a class $A^{*} X$.

Let $F \subset g^{*} V$ be a maximal isotropic subbundle. Then $c_{i}(F)(\bmod 2)$ is a Stiefel-Whitney class for $g^{*} V$. By Theorem 1,

$$
f^{\prime *} c_{i}(F) \equiv c_{i}\left(g^{*} E\right) \equiv g^{\prime *} c_{i}(E)(\bmod 2) \text {. }
$$

Now $F l(V) \rightarrow X$ is a proper Chow envelope, so by [K], Theorem 2.3] $c_{i}(E)$ is a pullback $(\bmod 2)$. 
To complete the proof we must show that for any $q: X^{\prime} \rightarrow X$, and any maximal isotropic subbundle $F \subset q^{*} V$, we have $q^{*} w_{2 i} \equiv c_{i}(F)(\bmod 2)$. To prove this consider the Cartesian diagram

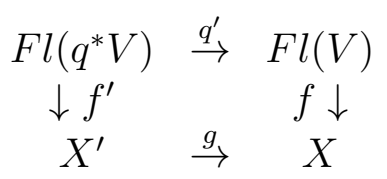

Chasing the diagram, and applying Theorem 1, it follows that $f^{\prime *} q^{*}\left(x_{n}\right)=$ $\pm f^{*}\left(c_{n}(F)\right)$. Since $f^{*}$ is injective $\bmod 2$, the existence of Stiefel-Whitney classes follows.

If $V$ has rank $2 n$, then we can prove the existence of Euler classes in $A^{n}(X)$ by the method above, using the fact that $c_{n}(E)= \pm c_{n}(F)$ for any two maximal isotropic subbundles. $\triangle$

Remark. If the bundle and quadratic form are not locally trivial in the Zariski topology it is still possible to construct (using our techniques) classes $\pm y_{n} \in A^{n}(X)$ (Theorem 3) such that $\pm \frac{y_{n}}{2^{n-1}}$ are Euler classes. On the other hand, the calculations of B. Totaro [T1] show that Stiefel-Whitney classes do not exist and that the Euler class is not integral for all quadratic vector bundles which are not locally trivial. In particular, he shows that on a variety approximating the classifying space $B S O(4)$ there is a tautological quadratic vector bundle where the topological Stiefel-Whitney and Euler classes are not represented by complex manifolds.

\section{Connections with topology}

In this section we explain the connection between the definitions of Euler and Stiefel-Whitney classes in the algebraic case and the usual definitions of these classes in topology. One consequence of this connection is a proof of a topological analogue of Theorem 11. Throughout this section, unless otherwise stated, we work in the setting of topology rather than algebraic geometry.

We begin by recalling a few facts about characteristic classes and classifying spaces. The space $B O(N, \mathbb{C})$ is the classifying space for rank $N$ complex vector bundles with nondegenerate quadratic form, and $B O(N, \mathbb{R})$ is the classifying space for rank $N$ real vector bundles. Let $\mathcal{V} \rightarrow B O(N, \mathbb{C})$ and 
$\mathcal{W} \rightarrow B O(N, \mathbb{R})$ be the universal vector bundles. The bundle $\mathcal{V}(\operatorname{resp} . \mathcal{W})$ has a nondegenerate (resp. positive definite real) quadratic form. Complexifying the quadratic form on $\mathcal{W}$ gives a nondegenerate quadratic form on $\mathcal{W} \otimes \mathbb{C}$. Because topologically the groups $O(N, \mathbb{C})$ and $O(N, \mathbb{R})$ are homotopy equivalent, we can identify the spaces $B O(N, \mathbb{C})$ and $B O(N, \mathbb{R})$. Under this identification, $\mathcal{V}=\mathcal{W} \otimes \mathbb{C}$, as bundles with quadratic form. Because this is the universal case, we have the following lemma.

Lemma 5 Let $V \rightarrow X$ be a complex vector bundle with nondegenerate quadratic form. Then there exists a real vector bundle $W \rightarrow X$ with positive definite quadratic form, such that $V \simeq W \otimes \mathbb{C}$ as vector bundles with quadratic form. Moreover, the classifying maps $X \rightarrow B O(N, \mathbb{C})$ of $V$ and $W$ coincide. $\triangle$

The cohomology ring $H^{*}(B O(N, \mathbb{C}) ; \mathbb{Z} / 2 \mathbb{Z})$ is isomorphic to the polynomial ring $\mathbb{Z} / 2 \mathbb{Z}\left[w_{1}, \ldots, w_{N}\right]$. The Stiefel-Whitney classes of a complex vector bundle $V$ with nondegenerate quadratic form (resp. real vector bundle $W$ ) over $X$ are defined to be the pullbacks of these classes via the classifying map to $H^{*}(X ; \mathbb{Z} / 2 \mathbb{Z})$. If the structure group of $V($ resp. $W)$ reduces to $S O(N, \mathbb{C})$ $($ resp. $S O(N, \mathbb{R}))$, then the classifying map $X \rightarrow B O(N, \mathbb{C})$ lifts (in exactly two ways, assuming $X$ connected) to a map $X \rightarrow B S O(N, \mathbb{C})$. If $N=2 n$, we single out a universal class $x_{n} \in H^{n}(B S O(2 n, \mathbb{C}) ; \mathbb{Z})$; the Euler classes of $V$ (resp. $W$ ) are defined to be the pullbacks of $x_{n}$ to $H^{n}(X ; \mathbb{Z})$ via the two lifts. These pullback classes differ only by sign.

If $V$ and $W$ are as in Lemma 5, then because the classifying maps of $V$ and $W$ coincide, their Stiefel-Whitney and Euler classes coincide as well.

Proposition 3 Let $V \rightarrow X$ be a complex vector bundle with nondegenerate quadratic form and let $E$ be a maximal isotropic subbundle of $V$. Then the even Stiefel-Whitney classes of $V$ are the mod 2 reductions of the Chern classes of $E$. Moreover, if the rank of $V$ is $2 n$, then the structure group of $V$ reduces to $S O(2 n, \mathbb{C})$, the Euler classes of $V$ are $\pm c_{n}(E)$, and the odd Stiefel-Whitney classes of $V$ vanish.

Proof: Choose $W$ as in Lemma 5 . Let $V_{\mathbb{R}}$ and $E_{\mathbb{R}}$ denote $V$ and $E$ viewed as real vector bundles. We have maps of real bundles

$$
E_{\mathbb{R}} \hookrightarrow V_{\mathbb{R}} \simeq W \oplus i W \rightarrow W
$$


The composition $E_{\mathbb{R}} \rightarrow W$ is injective, since $E$ is isotropic and the quadratic form on $W$ is positive definite. Assume now that the rank of $V$ is $2 n$. Then the ranks of $E_{\mathbb{R}}$ and $W$ are equal, so $E_{\mathbb{R}} \simeq W$. Since $W$ is isomorphic to the realification of a complex vector bundle, it is orientable as a real vector bundle, which means that the structure group of $W$ reduces to $S O(2 n, \mathbb{R})$. This implies that the structure group of $V$ reduces to $S O(2 n, \mathbb{C})$. The StiefelWhitney and Euler classes of $V$ equal those of $W$, which in turn equal those of $E_{\mathbb{R}}$. The assertions of the proposition then follow from Milnor-Stasheff, Problem 14-B and Definition, p. 158].

This proves the proposition in the even rank case. In the odd rank case, we can write $W \simeq E_{\mathbb{R}} \oplus L$, where $L$ is a real line bundle. The proposition then follows from the Whitney product formula and Milnor-Stasheff, Problem 14B]. $\triangle$

The preceding proposition shows that the Euler and Stiefel-Whitney classes we have defined in Chow cohomology bear the same relation to the Chern classes in Chow cohomology as the Euler and Stiefel-Whitney classes in topology do to the Chern classes in topology, and hence justifies our use of these names.

In the topological setting, the properties of Stiefel-Whitney and Euler classes given in Proposition 3 yield the following corollary, which is a topological analogue of Theorem 11. (In the algebraic case, the logic is reversed: we use Theorem 11 to prove the existence of Stiefel-Whitney and Euler classes.)

Corollary 1 Let $V \rightarrow X$ be a complex vector bundle with nondegenerate quadratic form, of rank $N$ equal to $2 n$ or $2 n+1$, and suppose that $E$ and $F$ are maximal isotropic subbundles of $V$. Then the $\bmod 2$ reductions of $c(E)$ and $c(F)$ are equal. Moreover, if $N=2 n$, then $c_{n}(E)= \pm c_{n}(F)$.

For smooth complex varieties the existence of a natural map from Chow cohomology to singular cohomology yields the following proposition.

Proposition 4 Let $X$ be a smooth complex variety and let $V \rightarrow X$ be a vector bundle with quadratic form, locally trivial in the Zariski topology. Then, under the natural map co: $A^{i}(X ; R) \rightarrow H^{2 i}(X ; R)$ (for $R=\mathbb{Z} / 2 \mathbb{Z}$ or $R=\mathbb{Z}$ ) the algebraic Stiefel-Whitney and Euler classes map to the corresponding topological classes. 
Proof: Both the algebraic and topological Stiefel-Whitney classes (resp. Euler classes) pull back to Chern classes of bundles on $F l(V)$. Since co maps algebraic Chern classes to topological Chern classes ([Fulton, Chapter 19]), the images of our algebraic classes pull back on $F l(V)$ to the pullbacks of the corresponding topological classes. The proposition then follows from the naturality of $c o$ and the following lemma.

Lemma 6 If $f: Y \rightarrow X$ is a proper Chow envelope of smooth complex varieties, then the pullback $f^{*}: H^{*}(X ; R) \rightarrow H^{*}(Y ; R)$ is injective for any coefficient group $R$.

Proof of Lemma 6: Consider the maps $c l: A_{i}(Y) \rightarrow H_{2 i}^{B M}(Y)$ and $c l$ : $A_{i}(X) \rightarrow H_{2 i}^{B M}(X)$ from Chow groups to Borel-Moore homology defined in [Fulton, Chapter 19]. Let $s=[\tilde{X}] \in A_{*}(Y)$, where $\tilde{X} \subset Y$ is a subvariety mapping birationally to $X$. Then $f_{*}(s)=[X]$. Since the class map is compatible with proper pushforward,

$$
f_{*}(\operatorname{cl}(s))=\operatorname{cl}([X])=\mu_{X},
$$

where $\mu_{X}$ is the fundamental homology class of $X$. If $x \in H^{*}(X)$, then by naturality of cap product,

$$
f_{*}\left(f^{*} x \cap c l(s)\right)=x \cap f_{*}(c l(s))=x \cap \mu_{X} .
$$

On the other hand, $-\cap \mu_{X}$ is the Poincare duality pairing (Fulton, Chapter 19]). Thus, if $f^{*} x=0 \in H^{*}(Y ; R)$, then the Poincare image of $x$ in $H_{*}^{B M}(X ; R)$ is zero. However, $X$ is smooth, so the pairing is perfect, and thus $x=0$. Therefore $f^{*}$ is injective. $\triangle$

\section{Chow rings of quadric and flag bundles with half integer coefficients}

In this section we study even rank bundles with quadratic form. We will not assume that bundle and its quadratic form are locally trivial in the Zariski topology. Instead, we assume, throughout this section, that the structure group reduces to $S O(2 n)$. We show (Theorem 3) that every such bundle $V \rightarrow X$ has characteristic classes $\pm y_{n} \in A^{n} X$, such that $\frac{y_{n}}{2^{n-1}}$ is an Euler 
class in $A^{*}\left(X ; \mathbb{Z}\left[\frac{1}{2}\right]\right)$. We use these Euler classes to compute the Chow rings tensored with $\mathbb{Z}\left[\frac{1}{2}\right]$ of the associated quadric and isotropic flag bundles. There is no discussion of the odd rank case because (over $\mathbb{Z}\left[\frac{1}{2}\right]$ ) the Chow groups of a quadric bundle are generated by powers of the hyperplane section.

Construction of the isotropic flag bundle as a tower of quadrics Let $V=V_{n}$ be a vector bundle of rank $2 n$ with a non-degenerate quadratic form. Let $Q=Q_{n-1} \rightarrow X$ be the corresponding quadric bundle. We have the following diagram:

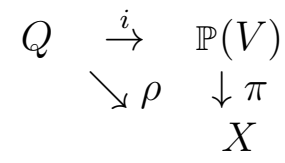

Let $V_{n}$ also denote the pullback bundle on $\mathbb{P}(V), S_{n}$ the tautological subbundle, and $S_{n}^{\perp}$ the orthogonal complement of $S_{n}$ in $V_{n}$. Although $S_{n}$ is not a subbundle of $S_{n}^{\perp}$, the pullback $i^{*} S_{n}$ is a subbundle of $i^{*} S_{n}^{\perp}$. Set $V_{n-1}=$ $i^{*} S_{n}^{\perp} / i^{*} S_{n}$; this is a rank $2 n-2$ vector bundle on $Q_{n-1}$ with nondegenerate quadratic form. Let $Q_{n-2} \rightarrow Q_{n-1}$ be the corresponding quadric bundle. In the same way, construct $V_{n-2}$, a rank $2 n-4$ vector bundle on $Q_{n-2}$. Continuing, we get a tower

$$
\begin{aligned}
& Q_{0} \rightarrow \mathbb{P}\left(V_{1}\right) \\
& \downarrow \\
& Q_{1} \rightarrow \mathbb{P}\left(V_{2}\right) \\
& \downarrow \\
& \ldots \rightarrow \mathbb{P}\left(V_{n-1}\right) \\
& \downarrow \\
& Q_{n-1} \quad \rightarrow \quad \mathbb{P}\left(V_{n}\right)=\mathbb{P}(V) \\
& \downarrow
\end{aligned}
$$

(There is an analogous tower in the odd rank case, but it is not essential to our discussion.)

Over $Q_{1}$ there is then a rank 2 vector bundle $V_{1}$ with nondegenerate quadratic form. The corresponding quadric bundle $Q_{0}$ is a double cover of $Q_{1}$. 
Lemma 7 With notation as above, if the structure group of $V_{n} \rightarrow X$ reduces to $S O(2 n)$, then the structure group of $V_{n-1} \rightarrow Q_{n-1}$ reduces to $S O(2 n-2)$. Moreover, if $V_{n}$ with its quadratic form is locally trivial in Zariski topology, then $V_{n-1}$ (with its quadratic form) is as well. $\triangle$

The proof is straightforward, and we omit it.

Since we are assuming that the structure group of $V_{n} \rightarrow X$ reduces to $S O(2 n)$, the above lemma implies that the structure group of $V_{1} \rightarrow Q_{1}$ reduces to $S O(2)$.

Lemma 8 Let $V_{1} \rightarrow X$ be a rank 2 vector bundle with nondegenerate quadratic form whose structure group reduces to $S O(2)$. Then $V$ splits into a direct sum $V_{1}^{\prime} \oplus V_{1}^{\prime \prime}$ of isotropic line bundles.

Proof: If $W$ is a vector space of rank 2 with a non-degenerate quadratic form, then $W$ has exactly 2 isotropic lines. These lines intersect in the origin, and each is invariant under $S O(2)$. Thus, if the transition functions of the bundle $V_{1}$ lie in $S O(2)$, we can construct two isotropic sub-linebundles of $V_{1}$ whose direct sum is $V$.

To avoid cumbersome notation, we will temporarily write $V_{i}, S_{i}$, etc. for the pullbacks of these bundles to $Q_{1}$.

The preceding lemma implies that the bundle $V_{1} \rightarrow Q_{1}$ splits into a direct sum $V_{1}^{\prime} \oplus V_{1}^{\prime \prime}$. This implies that each of the bundles $V_{2}, V_{3}, \ldots V_{n}$ has two isotropic subbundles when pulled back to $Q_{1}$. They can be defined as follows. Assume by induction that $V_{i-1}^{\prime}$ and $V_{i-1}^{\prime \prime}$, maximal isotropic subbundles of $V_{i}$ are defined. By definition we have a surjective map

$$
S_{i}^{\perp} \rightarrow S_{i}^{\perp} / S_{i}=V_{i-1} .
$$

Define $V_{i}^{\prime}\left(\right.$ resp. $\left.V_{i}^{\prime \prime}\right)$ to be the preimage of $V_{i-1}^{\prime}\left(\operatorname{resp} V_{i-1}^{\prime \prime}\right)$. Then $V_{i}^{\prime}$ and $V_{i}^{\prime \prime}$ are maximal isotropic subbundles of $V_{i}$. Note that by construction,

$$
c_{i}\left(V_{i}^{\prime}\right)=h_{i} c_{i-1}\left(V_{i-1}^{\prime}\right)
$$

where $h_{i}=c_{1}\left(S_{i}^{*}\right)$ is the hyperplane class on $\mathbb{P}\left(V_{i}\right)$.

The space $Q_{1}$ can be identified with the bundle $F l(V)$ of isotropic flags in $V$ of length $n-1$. On $F l(V)$ the bundle $V$ has a tautological flag of subbundles, which we will denote $E_{1} \subset E_{2} \subset \ldots \subset E_{n-1}$. In this notation, 
the bundles $V_{i}$ (pulled back to $F l_{n-1}$ ) are the quotients $E_{n-i}^{\perp} / E_{n-i}$ (taking $\left.E_{0}=0\right)$. The bundle $V$ has two maximal isotropic subbundles $V_{n}^{\prime}$ and $V_{n}^{\prime \prime}$. Using the fact that the bundles $V_{1}^{\prime}$ and $V_{1}^{\prime \prime}$ are dual, direct calculation, via Equation 1, shows that

$$
c_{n}\left(V_{n}^{\prime}\right)=-c_{n}\left(V_{n}^{\prime \prime}\right)
$$

Proposition 5 Let $f: F l\left(V_{n}\right) \rightarrow X$ be the projection. Then there is a (canonical) class $s \in A^{*}\left(F l\left(V_{n}\right)\right)$ such that for any $Y \rightarrow X$ and any $x \in$ $A^{*}(X), f_{*}\left(s \cdot f^{*} x \cap\left[F l\left(V_{n}\right) \times_{X} Y\right]\right)=2^{n-1} x \cap[Y]$. Hence $f^{*}$ is injective with $\mathbb{Z}\left[\frac{1}{2}\right]$ coefficients.

Proof: We identify $F l\left(V_{n}\right)=Q_{1}$. If $\rho_{i}: Q_{i-1} \rightarrow Q_{i}$ is a quadric in our tower, $x_{i} \in A^{*}\left(Q_{i}\right)$, and $Y \rightarrow Q_{i}$, then $\rho_{i *}\left(h_{i}^{2 i-2} \cdot \rho_{i}^{*} x \cap\left[Q_{i-1} \times_{X} Y\right]\right)=2 x \cap[Y]$. Thus $s=h_{2}^{2} h_{3}^{4} \ldots h_{n}^{2 n-2}$ (pulled back to $F l\left(V_{n}\right)$ ) is the desired class. $\triangle$

The following is an immediate consequence of Proposition 5 .

Theorem 3 Let $V$ be a vector bundle of rank $2 n$ with a non-degenerate quadratic form (not necessarily locally trivial in the Zariski topology) whose structure group reduces to $S O(2 n)$. Then there are characteristic classes $\pm y_{n} \in A^{n}(X)$ such that $\pm \frac{y_{n}}{2^{n-1}}$ are Euler classes in $A^{n}\left(X ; \mathbb{Z}\left[\frac{1}{2}\right]\right)$.

Remark: Theorem 3 strengthens a result of Vistoli $\mathbb{D}$ who showed (implicitly) the existence of an Euler class in $A^{*} X \otimes \mathbb{Q}$ for arbitrary principal $S O(2 n)$ bundles.

Proof: The existence of the class $s$ from Proposition 5 implies that if $c$ is in the kernel of the map $A^{*} F l(V) \stackrel{p_{1}^{*}-p_{2}^{*}}{\rightarrow} F l(V) \times_{X} F l(V)$ then the class $d$ in $A^{*} X$ defined by the formula

$$
d \cap[Y]=f_{*}\left(s \cdot c \cap\left[F l\left(V_{n}\right) \times_{X} Y\right]\right)
$$

satisfies $f^{*} d=2^{n-1} c$. This is an analogue of [K] Theorem 2.3] used above, and is proved in the same way. Setting $c=c_{n}(E)$ where $E$ is one of the two tautological maximal isotropic subbundles of $f^{*} V$, produces a class $y_{n} \in A^{*} X$ such that $f^{*} y_{n}=2^{n-1} c_{n}(E)$. The classes $\pm y_{n}$ are natural with respect to pullbacks, because the class $s$ of Proposition 5 is natural. 
Computation of Chow groups We now describe $A^{*}\left(Q ; \mathbb{Z}\left[\frac{1}{2}\right]\right)$ as an algebra over $A^{*}\left(X ; \mathbb{Z}\left[\frac{1}{2}\right]\right)$. Iterating over the quadric tower used above, we are also able to compute $A^{*}\left(F l(V) ; \mathbb{Z}\left[\frac{1}{2}\right]\right)$ as an algebra over $A^{*}\left(X ; \mathbb{Z}\left[\frac{1}{2}\right]\right)$.

Before we state the theorems, we need some further properties of Euler classes.

Proposition 6 Let $x_{n-1}$ be an Euler class for the associated bundle $V_{n-1} \rightarrow$ $Q_{n-1}$, and let $h$ be the pullback of the hyperplane section on $\mathbb{P}(V)=\mathbb{P}\left(V_{n}\right)$. Then $h x_{n-1}=\rho^{*} x_{n}$, where $x_{n} \in A^{n}\left(X ; \mathbb{Z}\left[\frac{1}{2}\right]\right)$ is an Euler class for $V$. If the bundle is locally trivial, then the identity holds integrally.

Proof: Let $f: F l(V) \stackrel{g}{\rightarrow} Q \stackrel{\rho}{\rightarrow} X$ be the projection. Then $f^{*} x_{n}=c_{n}\left(V_{n}^{\prime}\right)$ where $V_{n}^{\prime}$ is a maximal isotropic subbundle of $f^{*} V$. On the other hand, $g^{*} x_{n-1}=c_{n-1}\left(V_{n-1}^{\prime}\right)$ where $V_{n-1}^{\prime}$ is a maximal isotropic subbundle of $f^{*} V_{n-1}$. By Equation 1, $c_{n}\left(V_{n}^{\prime}\right)=h c_{n-1}\left(V_{n-1}^{\prime}\right)$, so the relation holds on $F l(V)$. Since $f^{*}$ is injective up to 2-torsion (or in the locally trivial case - integrally injective) the relation follows.

The following theorem is the key connection between Euler classes and Chow groups of quadric bundles. We use the same notation as Proposition 6 above.

Theorem 4 On a fiber of $Q_{n-1} \rightarrow X$ the Euler class $x_{n-1}$ restricts to $\pm\left(e_{n-1}-f_{n-1}\right)$, where $e_{n-1}$ and $f_{n-1}$ are the two ruling classes on the fiber.

Proof: Since we are working on a fiber, we may assume $X$ is a point, and $V=V_{n}$ is a vector space. Consider the tower above. The space $Q_{0}$ is the flag variety of length $n$ isotropic flags in $V$. It is a disconnected double cover of $Q_{1}=F l(V)$. Any element $g \in O(2 n)$ induces an automorphism of this tower. At each step denote the induced maps $Q_{i} \rightarrow Q_{i}$ and $\mathbb{P}\left(V_{i}\right) \rightarrow \mathbb{P}\left(V_{i}\right)$ by $g_{i}$. By construction, the $g_{i}$ 's are compatible for different values of $i$. If $g$ is not in the identity component of $O(2 n)$, then $g_{0}: Q_{0} \rightarrow Q_{0}$ acts by exchanging the two sheets of $Q_{0}$.

Lemma 9 Let $g$ be an element of $O(2 n)$ not in the identity component. Then $g_{1}^{*}\left(c_{i}\left(V_{i}^{\prime}\right)=-c_{i}\left(V_{i}^{\prime}\right)\right.$.

Proof of Lemma 9: It is clear that the hyperplane class in any of the projective bundles in the tower is invariant under pullback by the automorphism 
induced by $g$. Since $c_{i}\left(V_{i}^{\prime}\right)=h_{i} c_{i-1}\left(V_{i-1}^{\prime}\right)$, it suffices to prove the proposition when $i=1$. Now $Q_{0}$ is the union of two components, $\mathbb{P}\left(V_{1}^{\prime}\right)$ and $\mathbb{P}\left(V_{1}^{\prime \prime}\right)$, and $i_{0_{*}}\left(\left[\mathbb{P}\left(V_{1}^{\prime \prime}\right)\right]-\left[\mathbb{P}\left(V_{1}^{\prime}\right)\right]\right)=\pi_{1}^{*}\left(2 c_{1}\left(V_{1}^{\prime}\right)\right)$.

Since $g$ exchanges the two sheets of $Q_{0}$,

$$
g_{0}^{*}\left(\left[\mathbb{P}\left(V_{1}^{\prime}\right)\right]-\left[\mathbb{P}\left(V_{1}^{\prime \prime}\right)\right]\right)=-\left(\left[\mathbb{P}\left(V_{1}^{\prime}\right)\right]-\left[\mathbb{P}\left(V_{1}^{\prime \prime}\right)\right]\right) .
$$

Thus,

$$
g_{0}^{*}\left(\pi_{1}^{*}\left(2 c_{1}\left(V_{1}^{\prime}\right)\right)\right)=\pi_{1}^{*}\left(g_{1}^{*}\left(2 c_{1}\left(V_{1}^{\prime}\right)\right)\right)=-\pi_{1}^{*}\left(2 c_{1}\left(V_{1}^{\prime}\right)\right) .
$$

Since $\pi_{1}^{*}$ is injective, and we are working with coefficients in $\mathbb{Z}\left[\frac{1}{2}\right]$, the lemma follows.

Since $e_{n-1}$ and $f_{n-1}$ generate $A_{n-1}\left(Q_{n-1}\right)$ (Lemma 1), we can write $x_{n-1}=$ $a e_{n-1}+b f_{n-1}$, where $a$ and $b$ are in $\mathbb{Z}\left[\frac{1}{2}\right]$. Thus, $i_{*}\left(x_{n-1}\right)=(a+b) h_{n}$. Let $g$ be an element of $O(2 n)$ not in the identity component. Then in the notation of the preceding proposition, $g_{n-1}^{*} x_{n-1}=-x_{n-1}$. Thus $a+b=0$. Hence we can write $x_{n-1}=\lambda\left(e_{n-1}-f_{n-1}\right)$ for some $\lambda \in \mathbb{Z}\left[\frac{1}{2}\right]$. Since $x_{n-1}$ is an Euler class, $x_{n-1}^{2}=(-1)^{n} c_{2 n-2}\left(V_{n-1}\right)$ (Proposition 2). By direct calculation,

$$
c\left(V_{n-1}\right)=\frac{c\left(V_{n}\right)}{1-h_{n}^{2}} .
$$

But $c\left(V_{n}\right)=1$ since $V_{n}$ is trivial. Thus,

$$
x_{n-1}^{2}=(-1)^{n} c_{2 n-2}\left(V_{n-1}\right)=(-1)^{n} h_{n}^{2 n-2}=(-1)^{n} 2 .
$$

On the other hand, $x_{n-1}^{2}=\lambda^{2}\left(e_{n}-f_{n}\right)^{2}$, so by Lemma 2, $x_{n-1}^{2}=\lambda^{2} \cdot(-1)^{n} \cdot 2$. Therefore, $\lambda= \pm 1$ as desired. $\triangle$

Theorem 5 Let $X$ be a scheme and let $V \rightarrow X$ be a vector bundle of rank $2 n$ whose structure group reduces to $S O(2 n)$ with respect to a non-degenerate quadratic form on $V$. Let $Q \subset \mathbb{P}(V)$ be the associated quadric bundle. Then $A^{*}\left(Q ; \mathbb{Z}\left[\frac{1}{2}\right]\right)=A^{*}\left(X ; \mathbb{Z}\left[\frac{1}{2}\right]\right)\left[h, x_{n-1}\right] / I$, where $I$ is the ideal generated by the relations

$$
\begin{gathered}
h x_{n-1}=\rho^{*}\left(x_{n}\right) \\
x_{n-1}^{2}=(-1)^{n-1} c_{2 n-2}\left(V_{n-1}\right)=(-1)^{n-1}\left(h^{2 n-2}+h^{2 n-4} c_{2}(V)+\ldots+h^{2} c_{2 n-4}(V)+c_{2 n-2}(V)\right) .
\end{gathered}
$$


Remark: The relation

$$
h^{2 n}+h^{2 n-2} c_{2}(V)+\ldots+c_{2 n}(V)=0,
$$

inherited from $A^{*}(\mathbb{P}(V)) / A^{*}(X)$, can be easily derived from the two relations above. If $V \rightarrow X$ has rank $2 n+1$ then

$$
A^{*} Q=A^{*} X[h] / h^{2 n+1}+h^{2 n-1} c_{2}(V)+\ldots+c_{2 n}(V) .
$$

Example: Theorem 5 can be used to show that not all rank vector bundles of rank $N$ whose structure group reduces to $S O(N)$ have maximal isotropic subbundles. For an example when $N$ is even, let $Q_{2} \subset \mathbb{P}^{5}$ be a smooth quadric of rank 3. On $Q_{2}$ we can define the bundle $V_{2}=S^{\perp} / S$, where $S=\mathcal{O}(1)$, and $S^{\perp}$ is the orthogonal bundle with respect to the quadratic form on $Q$. Direct calculation shows that $V_{2}$ is locally trivial in the Zariski topology, so the bundle has rank 4 and structure group reduces to $S O(4)$. We claim, however, that $V_{2}$ has no isotropic subbundle of rank 2. Suppose to the contrary, that $E \subset V_{2}$ was such a bundle. Then $c_{2}(E)=x_{2}$, while $c_{1}(E)=a h$ for some constant $a$, since $h$ generates $A^{1}(Q)$. On the other hand,

$$
c(E) c\left(E^{*}\right)=c\left(V_{2}\right)=1+h^{2}+h^{4} .
$$

Thus,

$$
\left(1+a h+x_{2}\right)\left(1-a h+x_{2}\right)=1+h^{2}+h^{4} .
$$

In particular, $a^{2} h^{2}+2 x_{2}=h^{2}$, which is impossible since $h^{2}$ and $x_{2}$ are independent in $A^{2}(Q)$. Therefore, no such bundle $E$ exists. For an example of an odd rank bundle, let $V$ be the rank 3 bundle $S^{\perp} / S$ on the quadric hypersurface in $\mathbb{P}^{4}$. A similar calculation (using only the hyperplane class) shows that $V$ has no isotropic line subbundles.

Now let $f: F l(V) \rightarrow X$ be the flag bundle over $X$. Let $E_{1} \subset E_{2} \subset \ldots \subset$ $E_{n-1} \subset E_{n} \subset f^{*} V$ be one of the two tautological isotropic flags. Via the quadratic form, we can extend it to a complete flag $E_{1} \subset \ldots \subset E_{n} \ldots \subset$ $E_{2 n}=f^{*} V$. Set $h_{i}=c_{1}\left(E_{n-i+1} / E_{n-i}\right)\left(\right.$ where $\left.E_{0}=0\right)$.

Theorem 6 Let $V$ be as in the statement of Theorem 5. Then

$$
A^{*}\left(F l(V) ; \mathbb{Z}\left[\frac{1}{2}\right]\right)=A^{*}\left(X ; \mathbb{Z}\left[\frac{1}{2}\right]\right)\left[h_{1}, \ldots, h_{n}\right] / I
$$


where $I$ is the ideal generated the relations

$$
\begin{gathered}
\prod_{i=1}^{n}\left(1-h_{i}^{2}\right)=f^{*} c(V) \\
h_{1} h_{2} \ldots h_{n}=f^{*} x_{n}
\end{gathered}
$$

Remark: The choice of notation $h_{i}$ in the above theorem is consistent with its use previously, since for $i>1, c_{1}\left(E_{n-i+1} / E_{n-i}\right)$ is the pullback of the hyperplane class of the projective bundle $\mathbb{P}\left(V_{i}\right)$.

Proofs of Theorems 5 and 6: We give the proof assuming $X$ is smooth to avoid using the language of bivariant intersection theory ( Fulton, Chapter 17]). A reader familiar with this theory can easily extend the proof to singular schemes.

Theorem 6 follows from Theorem 5 by iterating over the tower of quadrics constructed previously. Thus it suffices to prove Theorem 5. First note that $A^{*} X\left[h, x_{n}\right] / I$ has a basis of monomials $h^{i} x_{n}^{\alpha}$, where $0 \leq i \leq n-1$, and $\alpha \in\{0,1\}$. Since $x_{n-1}$ restricts on a fiber to the difference of two rulings, these classes restrict to a basis for the Chow group tensored with $\mathbb{Z}\left[\frac{1}{2}\right]$ of a fiber (Lemma 1). At this point we would like to apply Noetherian induction and conclude that $h^{i} x_{n}^{\alpha}$ is a basis for the Chow groups of $Q$ over $A^{*}(X)$. Unfortunately, the bundle $Q \stackrel{\rho}{\rightarrow} X$ need not be locally trivial in the Zariski topology. However, $Q \times_{X} F l(V)$ is Zariski locally trivial over $F l(V)$ since the pullback of $V$ has a maximal isotropic subbundle. Thus, by Noetherian induction, our classes pull back to a basis for $A^{*}\left(Q \times_{X} F l(V) ; \mathbb{Z}\left[\frac{1}{2}\right]\right)$ over $A^{*}(F l(V))$. On the other hand, the pullback $f^{*}: A^{*}\left(X ; \mathbb{Z}\left[\frac{1}{2}\right]\right) \rightarrow A^{*}\left(F l(V) ; \mathbb{Z}\left[\frac{1}{2}\right]\right)$ has a section (see Proposition 5). Pushing forward via the section, we conclude that $\left\{h^{i} x_{n}^{\alpha}\right\}$ is a basis for $A^{*}\left(Q ; \mathbb{Z}\left[\frac{1}{2}\right]\right)$.

To complete the proof, we must check the relations. The relation $h x_{n-1}=$ $\rho^{*}\left(x_{n}\right)$ is Proposition 6. Since $x_{n-1}$ is an Euler class for the bundle $V_{n-1}$, $x_{n-1}^{2}=(-1)^{n-1} c_{2 n-2}\left(V_{n-1}\right)$ (Proposition 2). Since $c_{2 n-2}\left(V_{n-1}\right)=\left\{\frac{c(V)}{1-h^{2}}\right\}_{2 n-2}$, the second relation follows. $\triangle$

Remark: In general $A^{*}(F l(V))$ need not be free over $A^{*}(X)$. If it were, then one could define an integral Euler class for bundles whose structure group reduces to $S O(2 n)$, but were not locally trivial in the Zariski topology. 
However, we noted before that B. Totaro ([T1]) has shown that this can not be true.

Remark: If the stucture group $V \rightarrow X$ does not reduce to $S O(2 n)$, then the flag variety $f: F l_{n}(V) \rightarrow X$ of length $n$ isotropic flags is connected. If $E \subset f^{*} V$ is a tautological rank $n$ isotropic subbundle, then arguments similar to those used in Lemma 9 shows that $c_{n}(E)=0 \in A^{*}\left(F l_{n}(V) ; \mathbb{Z}\left[\frac{1}{2}\right]\right)$. Thus the Euler class is also 2-torsion, a fact consistent with topology, since non-orientable bundles only have Thom classes with $\mathbb{Z} / 2 \mathbb{Z}$ coefficients. This calculation also shows that $c_{2 n}(V)$ is 2 -torsion as well.

\section{Chow groups of quadric and isotropic flag bundles with integer coefficients}

Let $V \rightarrow X$ be a vector bundle with a quadratic form, and let $F \subset V$ be a fixed maximal isotropic subbundle. The purpose of this section is to compute the Chow rings of the associated quadric and flag bundles. The presentation will depend on the particular subbundle $F$.

\section{Chow rings of quadric bundles}

Theorem 7 Let $V$ be a vector bundle of rank $N$ with with a nondegenerate quadratic form on a scheme $X$. Let $Q \stackrel{\rho}{\rightarrow} X$ be the associated quadric bundle and $h$ the hyperplane class on $Q$. Assume that $F \subset V$ is a maximal isotropic subbundle and set $\gamma=[\mathbb{P}(F)] \subset Q$. Then

$$
A^{*}(Q)=A^{*} X[h, \gamma] / I
$$

where $I$ is the ideal generated by the relations

$$
\begin{aligned}
& 2 h \gamma=h^{n}-c_{1}(F) h^{n-1}+\ldots+(-1)^{n} c_{n}(F) \\
& \gamma^{2}=(-1)^{n-1}\left(c_{n-1}(F)+c_{n-3}(F) h^{2}+\ldots\right) \gamma
\end{aligned}
$$

if $N=2 n$, and the relations

$$
\begin{aligned}
2 h \gamma & =h^{n+1}+c_{1}(V / F) h^{n}+\ldots c_{n+1}(V / F) \\
\gamma^{2} & =\left(c_{n}(V / F)+c_{n-2}(V / F) h^{2}+\ldots\right) \gamma
\end{aligned}
$$

if $N=2 n+1$. 
Proof: We give the proof only in the even case, as the odd case is analogous. As in the proof of Theorem 3 we assume $X$ is smooth. A basis for $A^{*} X[h, \gamma] / I$ as an $A^{*} X$ module is the monomials $1, h, h^{2}, \ldots h^{n-1}, \gamma, h \gamma, \ldots, h^{n-1} \gamma$. These classes restrict to a basis of the Chow groups of the fiber, so they form a basis for $A^{*}(F l(V))$ as a $A^{*}(X)$ module (All bundles are locally trivial in Zariski topology, so we can apply Noetherian induction). To prove the theorem we must check the relations.

Let $i: Q \hookrightarrow \mathbb{P}(V)$ be the inclusion. From the proof of Theorem 1, we know that $i_{*} \gamma=H^{n}-c_{1}(F) H^{n-1}+\ldots+(-1)^{n} c_{n}(F)$, where $H$ is the hyperplane section on $\mathbb{P}(V)$. Hence the right hand side of the first relation is $i^{*} i_{*} \gamma$. On the other hand, the normal bundle to $Q$ in $\mathbb{P}(V)$ is $2 h$. Thus, by the self intersection formula, $i^{*} i_{*} \gamma=2 h \gamma$. This proves the first relation.

The second relation follows from the self intersection formula $\gamma^{2}=j_{*}\left(c_{n-1}\left(N_{\mathbb{P}(F)} Q\right)\right) \gamma$ where $j_{*}: \mathbb{P}(F) \hookrightarrow Q$ is the inclusion. The normal bundle is

$$
N_{\mathbb{P}(F)} Q=N_{\mathbb{P}(F)} \mathbb{P}(V) / N_{Q} \mathbb{P}(V)=\frac{V / F \otimes S^{*}}{\left(S^{*}\right)^{\otimes 2}} .
$$

Tensoring the righthand side with $S \otimes S^{*}$, and identifying $V / F$ with $F^{*}$ we can identify the normal bundle as $\frac{F^{*}}{S^{*}} \otimes S^{*}$. The formula then follows by direct calculation. $\triangle$

Splitting bundles with quadratic form Let $V=L_{1} \oplus L_{2} \oplus \ldots \oplus L_{N}$ be a direct sum of line bundles of the form $L_{1} \oplus L_{2} \oplus \ldots \oplus L_{n} \oplus L_{n}^{*} \oplus \ldots \oplus L_{1}^{*}$ if $N=2 n$, and $L_{1} \oplus \ldots \oplus L_{n} \oplus M \oplus L_{n}^{*} \oplus \ldots \oplus L_{1}$, if $N=2 n+1$, where $M$ is a self-dual line bundle. Define a quadratic form $q_{s t d}$ on $V$ by the rule that $q_{s t d}$ restricted to $L_{i} \oplus L_{N-i+1}$ is the canonical pairing, and $L_{i}$ is orthogonal to $L_{j}$ otherwise. We will call $q_{\text {std }}$ the standard quadratic form on $V$. Suppose that $q$ is a quadratic form on $V$ such that $q$ restricted to $L_{i} \oplus L_{N-i+1}$ is the canonical pairing and such that (with respect to $q$ ), $L_{i}$ is orthogonal to $L_{1}, \ldots, L_{N-i}$.

Lemma 10 Let $V, q$ and $q_{\text {std }}$ be as above. Then, (assuming that the base field has characteristic not equal to 2), there exists a vector bundle automorphism $g$ of $V$ such that $g$ is the identity on $L_{1} \oplus \ldots L_{n}$ and such that $g^{*} q_{s t d}=q$. 
The proof of this lemma is an exercise in "bundle-ized" linear algebra which we omit.

Remark: Fulton [F1] shows that there is a deformation of $(V, q)$ to $\left(V, q_{s t d}\right)$ that induces an isomorphism of the Chow rings of the associated isotropic flag bundles. As a result, Theorems 8 and 9 are still valid in characteristic 2 .

The following quadratic splitting principle (in characteristic not equal to 2 ) is an application of the lemma above.

Proposition 7 Let $V \rightarrow X$ be a vector bundle of $\operatorname{rank} N=2 n$ or $N=2 n+1$ with a nondegenerate quadratic form. Let $E_{1} \subset \ldots E_{n}$ be a maximal isotropic flag of subbundles of $V$. Set $L_{i}=E_{i} / E_{i-1}$ and if $N$ is odd, set $M=E_{n}^{\perp} / E_{n}$. Then there exists $f: Y \rightarrow X$ with $f^{*}: A^{*} X \rightarrow A^{*} Y$ injective, and an isomorphism of $f^{*} V$ with $L_{1} \oplus \ldots L_{n} \oplus L_{n}^{*} \oplus \ldots \oplus L_{1}^{*}$ or $L_{1} \oplus \ldots L_{n} \oplus M \oplus$ $L_{n}^{*} \ldots \oplus L_{1}^{*}$, as vector bundles with quadratic form ( where $f^{*} V$ is given the quadratic form inherited from $V$, and the direct sum of line bundles is given the standard form described above). Furthermore, the subbundle $E_{i}$ corresponds to the sum $L_{1} \oplus \ldots \oplus L_{i}$.

Proof: Extend $E_{1} \subset \ldots \subset E_{n}$ to a complete flag $E_{1} \subset \ldots \subset E_{N}=V$ by setting $E_{n+i}=E_{n-i}^{\perp}$. Then $L_{n+i}=E_{n+i} / E_{n+i-1} \simeq L_{n-i+1}^{*}$. Then the complete splitting principle as stated in [F1] (cf. [G, Theorem 8.3]) gives $f: Y \rightarrow X$ with $f^{*}$ injective and and isomorphism of $f^{*} V$ with $L_{1} \oplus \ldots L_{n} \oplus$ $L_{n}^{*} \oplus L_{1}^{*}$ or $L_{1} \oplus \ldots L_{n} \oplus M \oplus L_{n}^{*} \ldots \oplus L_{1}^{*}$, as vector bundles. Let $q$ be the quadratic form on $L_{1} \oplus \ldots \oplus L_{N}$ pulled back by isomorphism from the quadratic form on $f^{*} V$. By construction, $q$ restricts to the dual pairing on $L_{i} \oplus L_{N-i+1}$. Moreover, since we began with a maximal isotropic flag, each $L_{i}$ is orthogonal to $L_{1}, \ldots L_{N-i}$. By the above lemma, there exists a vector bundle automorphism of $L_{1} \oplus \ldots \oplus L_{N}$ taking $q$ to $q_{s t d}$. This proves the proposition. $\triangle$

Presentation of the Chow rings of flag bundles Let $V$ be a vector bundle with a nondegenerate quadratic form on a scheme $X$. We assume the existence of a maximal isotropic flag $F .=F_{1} \subset F_{2} \ldots \subset F_{n} \subset V$. To compute the Chow ring we need only assume the existence of a maximal isotropic subbundle $F$. However, assuming the existence of the full flag $F$. gives a presentation consistent with the Schubert variety ideas of E1. 
Let $\pi: F l(V) \rightarrow X$ be the bundle of maximal isotropic flags in $V$. (Recall that if the rank $V$ is $2 n$, then the flag variety has two isomorphic connected components. In this case the notation $F l(V)$ will refer to one of these components, which may be identified with $F l_{n-1}(V)$.) Set $y_{i}=$ $-c_{1}\left(\pi^{*} F_{i} / \pi^{*} F_{i-1}\right)$. The following two theorems (depending on whether the rank of $V$ is even or odd) describe $A^{*} F l(V)$ as an algebra over $A^{*} X$.

Theorem 8 ( $D_{n}$ case): If $V$ has rank $2 n$ then $A^{*} F l(V)=A^{*} X\left[x_{1}, x_{2}, \ldots, x_{n}, c_{1}, \ldots c_{n-1}\right] / I$ where $I$ is the ideal generated by the following relations (for $1 \leq i \leq n$ ):

$$
\begin{aligned}
& e_{i}\left(-x_{1}^{2}, \ldots,-x_{n}^{2}\right)=\pi^{*} c_{2 i}(V) \\
& (-1)^{p} c_{p}^{2}+(-1)^{p-1} 2 c_{p-1} c_{p+1}+\ldots-2 c_{2 p-1} c_{1}+2 c_{p}=c_{2 p}-c_{2 p-1} e_{1}\left(x_{1}, \ldots x_{n}\right)+\ldots+(-1)^{p} c_{p} e_{p}\left(x_{1}, \ldots, x_{n}\right) \\
& 2 c_{i}=e_{i}\left(x_{1}, \ldots, x_{n}\right)+e_{i}\left(y_{1}, \ldots, y_{n}\right) \\
& \left(c_{k}=0 \text { for } k \geq n\right) .
\end{aligned}
$$

Recall that if $a_{1}, \ldots a_{n}$ is a set of variables then $e_{i}\left(a_{1}, \ldots, a_{n}\right)$ denotes the $i$-th elementary symmetric polynomial in those variables.

Theorem 9 ( $B_{n}$ case): If $V$ has rank $2 n+1$, then $A^{*} F l(V)=A^{*} X\left[x_{1}, \ldots, x_{n}, c_{1}, \ldots, c_{n}\right] / I$ where $I$ is the ideal generated by the following relations (for $1 \leq i \leq n$ )

$$
\begin{gathered}
e_{i}\left(-x_{1}^{2}, \ldots,-x_{n}^{2}\right)=\pi^{*} c_{2 i}(V) \\
l e_{i}\left(-x_{1}^{2}, \ldots,-x_{n}^{2}\right)=\pi^{*} c_{2 i+1}(V) \\
2 c_{i}=e_{i}\left(x_{1}+l, \ldots, x_{n}+l\right)+e_{i}\left(y_{1}+l, y_{2}+l, \ldots, y_{n}+l\right) \\
(-1)^{p} c_{p}^{2}+(-1)^{p-1} 2 c_{p-1} c_{p+1}+\ldots-2 c_{2 p-1} c_{1}+2 c_{p} \\
=c_{2 p}-c_{2 p-1} e_{1}\left(x_{1}+l, \ldots x_{n}+l\right)+\ldots+(-1)^{p} c_{p} e_{p}\left(x_{1}+l, \ldots, x_{n}+l\right)
\end{gathered}
$$

where $l=\pi^{*} c_{1}(V)$

Proof of Theorem 8. If $V$ has rank 2n, let $E_{1} \subset E_{2} \ldots \subset E_{n}$ be a tautological flag on $\pi^{*} V$ such that $E_{n}$ and $\pi^{*} F_{n}$ are in opposite ruling families. Set $x_{i}=c_{i}\left(E_{i} / E_{i-1}\right)$. Since $\pi^{*} V / E_{n}=E_{n}^{*}, e_{i}\left(-x_{1}^{2}, \ldots-x_{n}^{2}\right)=c_{2 i}\left(\pi^{*} V\right)$ proving the first relation. 
Let $\rho: Q \rightarrow F l(V)$ be the quadric bundle associated to $\pi^{*} V$. Define classes $c_{i} \in A^{*}(F l(V))$ by the relation

$$
\left[\mathbb{P}\left(E_{n}\right)\right]+\left[\mathbb{P}\left(\pi^{*} F_{n}\right)\right]=h^{n-1}-h^{n-2} \rho^{*} c_{1}+h^{n-3} \rho^{*} c_{2}-\ldots+(-1)^{n-1} \rho^{*} c_{n-1}
$$

Since $\rho_{*}\left(h^{n}\left[\mathbb{P}\left(E_{n}\right)\right] \rho^{*} c_{i}\right)=c_{i}$, the $c_{i}$ 's are uniquely determined. Furthermore, by the proof of Theorem 1, $2 c_{i}=c_{i}\left(E_{n}\right)+c_{i}\left(F_{n}\right)=e_{i}\left(x_{1}, \ldots, x_{n}\right)+$ $e_{i}\left(y_{1}, \ldots, y_{n}\right)$.

Note that $A^{*} X\left[x_{1}, \ldots x_{n}, c_{1}, \ldots c_{n-1}\right] / I$ has a basis of monomials of the form

$$
x_{1}^{a_{1}} x_{2}^{a_{2}} \cdots x_{n}^{a_{n}} c_{1}^{\alpha_{1}} c_{2}^{\alpha_{2}} \cdots c_{n-1}^{\alpha_{n-1}}
$$

where $0 \leq a_{i} \leq n-i$ and $\alpha_{i} \in\{0,1\}$. On the other hand, Fulton [F1] (generalizing a result stated in $\mathbb{M}$ ) has shown that these monomials form a basis for $A^{*}(F l(V))$ as an $A^{*} X$ module. Therefore, to prove the theorem it suffices to check that the second relation holds.

By Proposition 7 there is a map $f: Y \rightarrow F l(V)$ such that $f^{*}$ is injective and $\pi^{*} V$ pulls back to $L_{1} \oplus L_{2} \ldots L_{n} \oplus L_{n}^{*} \oplus \ldots \oplus L_{1}$ with the standard quadratic form. To check a relation, it suffices to check it for the totally split bundle on $Y$.

Furthermore, there is always a map $g: Y^{\prime} \rightarrow Y$ with $g^{*}$ injective such that $Y^{\prime}$ is quasi-projective (this is Chow's Lemma combined with Nagata's embedding theorem, see [Fulton, Section 18.3]). We may therefore assume that $Y$ is quasi-projective. In particular there are maps $j_{i}: Y \rightarrow \mathbb{P}^{k_{i}}$ such that $L_{i}=j_{i}^{*} M_{i}$ for some line bundle $M_{i}$ on $\mathbb{P}^{k_{i}}$. Setting $Z=\mathbb{P}^{k_{1}} \times \mathbb{P}^{k_{2}} \times \ldots \times \mathbb{P}^{k_{n}}$, there is a map $j: Y \rightarrow Z$ such $L_{1} \oplus L_{2} \oplus \ldots L_{n} \oplus L_{n}^{*} \oplus \ldots \oplus L_{n}^{*}$ is the pullback of direct sum of line bundles and their duals on $Z$. To prove the relation, it suffices to check it in $A^{*} Z$. However, since $A^{*} Z$ is torsion free, we need only check that the relation holds up to multiplication by a constant. Since $2 c_{p}=e_{p}\left(x_{1}, \ldots, x_{n}\right)+e_{p}\left(y_{1}, \ldots y_{n}\right)$, multiplying both sides of the potential relation by 4 we reduce to showing that the degree $2 p$ term in

$$
\left(c\left(E_{n}\right)+c\left(\pi^{*} F_{n}\right)\right)\left(c\left(E_{n}^{*}\right)+c\left(\pi^{*} F_{n}^{*}\right)\right)
$$

equals the degree $2 p$ term in

$$
c\left(E_{n}\right)\left(c\left(E_{n}^{*}\right)+c\left(\pi^{*} F_{n}\right)\right)+c\left(E_{n}^{*}\right)\left(c\left(E_{n}\right)+c\left(\pi^{*} F_{n}\right)\right) .
$$

This equality is immediate since

$$
c\left(E_{n}\right) c\left(E_{n}^{*}\right)=c\left(\pi^{*} F_{n}\right) c\left(\pi^{*} F_{n}^{*}\right)=c\left(\pi^{*} V\right) .
$$


This proves Theorem 8 . $\triangle$

Proof of Theorem 9. If $V$ has rank $2 n+1$, let $E_{1} \subset E_{2} \subset \ldots \subset E_{n}$ be the tautological isotropic flag on $F l(V)$. Again set $x_{i}=c_{1}\left(E_{i} / E_{i-1}\right)$. Let $L=E_{n+1} / E_{n}$. Then $l=c_{1}\left(\pi^{*} V\right)$, and $c(V)=c(E) c\left(E^{*}\right)(1+l)$. This shows that the first two relations hold.

Again let $\rho: Q \rightarrow F l(V)$ be the quadric bundle associated to $\pi^{*}(V) \otimes L$. Define the $c_{i}$ by the equation

$$
\left[\mathbb{P}\left(E_{n} \otimes L\right)+\mathbb{P}\left(\pi^{*} F_{n} \otimes L\right)\right]=h^{n}-h^{n-1} \rho^{*} c_{1}+h^{n-1} \rho^{*} c_{2}-\ldots+(-1)^{n} \rho^{*} c_{n} .
$$

The proof of Theorem 11 also shows that $2 c_{i}=c_{i}\left(E_{n} \otimes L\right)+c_{i}\left(\pi^{*} F_{n} \otimes L\right)$, thereby proving the last relation.

Fulton [F1] has also shown that the appropriate monomials generate $A^{*}(F l(V))$ as an $A^{*}(X)$ module, so that proving the theorem reduces to checking the quadratic relation on the $c_{i}$ 's.

The proof of the quadratic relation in the $B_{n}$ case is more or less the same as that given above. There is one twist. The totally split pullback of $\pi^{*} V$ is $L_{1} \oplus L_{2} \oplus \ldots L_{n} \oplus M \oplus L_{n}^{*} \oplus \ldots \oplus L_{1}^{*}$, where $M$ is self dual. However, $\pi^{*}(V) \otimes M$ totally splits as $M_{1} \oplus M_{2} \ldots \oplus M_{n} \oplus \mathcal{O} \oplus M_{n}^{*} \oplus \ldots \oplus M_{1}^{*}$ (where $\left.M_{i}=L_{i} \otimes M\right)$. We can then reduce to checking the relation on a product of projective spaces. The relation on the $c_{p}$ 's then follows from the fact that

$$
c\left(E_{n} \otimes M\right) c\left(E_{n}^{*} \otimes M\right)=c\left(\pi^{*} F_{n} \otimes M\right) c\left(\pi^{*} F_{n}^{*} \otimes M\right) .
$$

This proves Theorem 9. $\triangle$

\section{References}

[EG] D. Edidin, W. Graham, Characteristic classes in the Chow ring preprint.

[F1] W. Fulton, Schubert varieties in flag bundles, preprint 1994.

[F2] W. Fulton, Determinantal formulas for orthogonal and symplectic degeneracy loci, preprint 1994.

[Fulton] W. Fulton, Intersection Theory, Ergebnisse, 3. Folge, Band 2, Springer Verlag, (1984). 
[G] H. Gillet, Riemann-Roch theorems for higher K-theory, Adv. Math. 40 (1981), 203-289.

[K] S. Kimura, Fractional intersection theory and bivariant theory, Comm. Alg. 20 (1992) 285-302.

[M] R. Marlin, Anneaux de Chow des groupes algebraiques $S U(n), S p(n), S O(n), \operatorname{Spin}(n), G_{2}, F_{4}$; torsion, C.R. Acad Sc. Paris, Series A, 279 (1974), 119-122.

[Milnor-Stasheff] J. Milnor, J. Stasheff, Characteristic Classes, Princeton University Press (1974).

[T1] B. Totaro, preprint 1994.

[T2] B. Totaro, preprint 1994.

[Sem-Chev] Anneau de Chow et applications, Seminaire Chevalley, Secrétariat mathématique, Paris (1958).

[S] R. Swan , K-Theory of quadric hypersurfaces, Annals of Math 122 (1985), 113-153.

[V] A. Vistoli, Characteristic classes of principal bundles in algebraic intersection theory, Duke Math J. 58 (1989), 299-315. 